\title{
Luminosity Distribution of Gamma-ray Burst Optical Afterglows $\dagger$
}

\author{
X. G. Wang ${ }^{1}$, E. W. Liang ${ }^{1,2}$, L. Li ${ }^{1}$, J. J. Wei ${ }^{1}$, B. Zhang ${ }^{3,1}$ \\ ${ }^{1}$ Department of Physics and GXU-NAOC Center for Astrophysics and Space Sciences, \\ Guangxi University, Nanning, 530004, China \\ email: lew@gxu.edu.cn \\ ${ }^{2}$ National Astronomical Observatories, Chinese Academy of Sciences, Beijing, 100012, China \\ ${ }^{3}$ Department of Physics and Astronomy, University of Nevada, Las Vegas, NV 89154,USA
}

\begin{abstract}
We derive the optical afterglow luminosity distributions at different epoches for gamma-ray bursts (GRBs) from a sample of 146 GRBs that have a well-sampled optical afterglow lightcurve, then explore the luminosity function of GRB optical afterglows using the Monte Carlo simulation. We show that an intrinsic broken power-law luminosity function can well reproduced the observed magnitude distributions.
\end{abstract}

Keywords. Gamma-Ray Burst, luminosity function, Monte Carlo simulation

\section{Introduction}

The optical afterglows are promising probes for the properties of high- $z$ galaxies (e.g., Liang et al. 2006; Savaglio et al. 2009). However, the optical afterglow luminosity function (LF) and the nature of optically dark gamma-ray bursts (GRBs) are quit uncertain, although the number of GRBs with optical afterglow detection rapidly increases since the launch of the Swift mission. It is noted that the observed afterglow emission is a superposition of the traditional external shock afterglow and an afterglow related to the late central engine activities. We have present a detailed analysis of the optical lightcurves in order to decompose the emission components from the lightcurves and investigate their implications ( $\mathrm{Li}$ et al. 2012; Liang et al. 2012). This paper dedicate to present the observed luminosity distributions of optical afterglows at different epoches and explore the luminosity functions of the optical afterglows.

\section{Observed Optical Brightness distributions}

We compile well-sampled optical afterglow lightcurves for 146 Gamma-Ray Bursts from literature. The k-correction and Galactic extinction correction have been made. We fit the lightcurves with a model of multiple components. The details of the sample and our lightcurve fits please refer to Li et al. (2012) and Liang et al. (2012). We derive the luminosity of each component at different epoches from our lightcurve fits. We find that observed R-band magnitude of the traditional afterglow component usually $\sim 15.7$ and it is $\sim 16.8$ at the end of the shallow decay segment. Figure 1 shows the R-Band magnitude distributions at $100 \mathrm{~s}$ and $1000 \mathrm{~s}$ since the GRB trigger from the global lightcurves, which

$\dagger$ Supported by the "973" Program of China (2009CB824800), the National Natural Science Foundation of China (Grants No. 11025313), and Special Foundation for Distinguished Expert Program of Guangxi, the Guangxi Natural Science Foundation (2010GXNSFA013112, 2011GXNSFB018063 and 2010GXNSFC013011), the special funding for national outstanding young scientist (Contract No. 2011-135), and the 3th Innovation Projet of Guangxi University. 

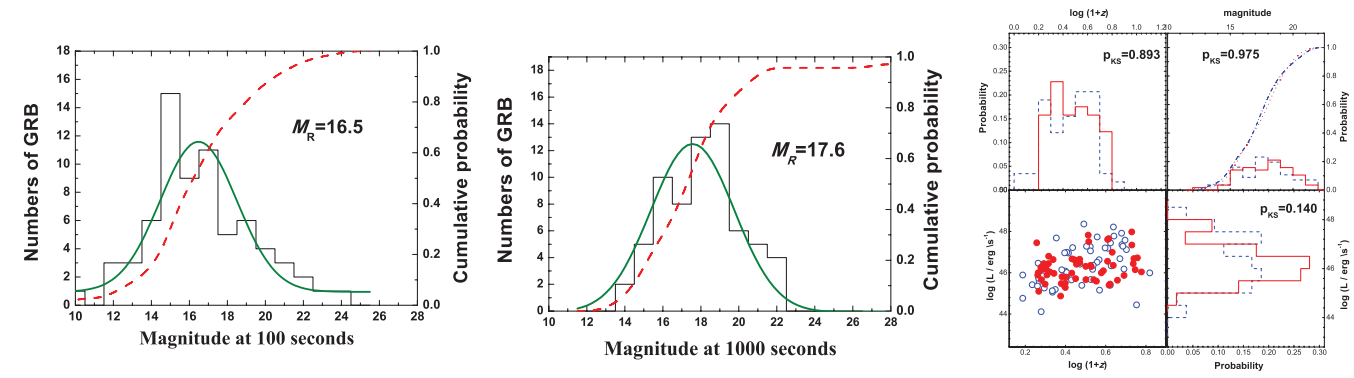

Figure 1. R band magnitude distributions of global lightcurves in different epoches, and our simulated $L-z$ distribution for $t=1000 \mathrm{~s}$. The blue dots and lines are for observational data and the red symbols are of simulations.

are the sum of the luminosities of all components. They roughly can be fit with a Gaussian function, which yields center a value of $M_{R} \sim 16.5$ at 100 seconds and 17.6 at $10^{3} \mathrm{~s}$.

\section{MC Simulations}

The observed optical luminosity suffers great instrumental biases and host galaxy extinctions. We investigate the intrinsic LF of various optical emission components via MC simulations. A broke-power-law function is adopted to describe the intrinsic LF for all components, which reads $\Phi(L, z)=\Phi_{0}\left[\left(\frac{L}{L_{\mathrm{b}}}\right)^{\alpha_{1}}+\left(\frac{L}{L_{\mathrm{b}}}\right)^{\alpha_{2}}\right]^{-1}$. We assume that the GRB Rate follows the star formation history and matellicity history. The evolution of $L_{b}$ is not considered in our analysis. We simulate a large sample of GRBs that can be triggered with Swift/BAT. The optical flux limit of the afterglows is adopted as $M_{R}=20$. The host galaxy extinction distribution is from current sample. The details of the simulation method please refer to Qin et al. (2010) and Lu et al. (2012). We use the probability from the K-S tests to estimate the consistency between our simulation results and observations. Our simulation results are also shown in Figure 1. Our best fit parameter set is $\left\{\alpha_{1}, \alpha_{2}, L_{\mathrm{b}}\right\}=\left\{0.70,1.9, \sim 10^{46} \mathrm{erg} \mathrm{s}^{-1}\right\}$.

\section{Summary}

We have derived the luminosity distributions of the optical afterglow at different epoches. We show that the R-Band magnitude distributions at $100 \mathrm{~s}$ and $1000 \mathrm{~s}$ of the global lightcurves are roughly Gaussian, which centers at $M_{R} \sim 16.5$ at 100 seconds and 17.6 at $10^{3} \mathrm{~s}$. Our MC simulations suggest that an intrinsic broken power-law luminosity function with a parameter set $\left\{\alpha_{1}, \alpha_{2}, L_{\mathrm{b}}\right\}=\left\{0.70,1.9, \sim 10^{46} \mathrm{erg} \mathrm{s}^{-1}\right\}$. can well reproduced the observed magnitude distributions.

\section{References}

Li, L., Liang, E. W., Tang, Q. W., et al. 2012, ApJ, 758, 27

Liang, E. W. \& Zhang, B. 2006, ApJ, 638, L67

Liang, E. W., Li, L., He, G., et al. 2012, ApJ, submitted

Lu, R. J., Wei, J. J., Qin, S. F., \& Liang, E. W. 2012, ApJ, 745, 168

Qin, S. F., Liang, E. W., Lu, R. J., et al. 2010, MNRAS, 406, 558

Savaglio, S., Glazebrook, K., \& Le Borgne, D. 2009, ApJ, 691, 182 